\title{
FACT - Influence of SiPM Crosstalk on the Performance of an Operating Cherenkov Telescope
}

\author{
J. Buß ${ }^{* b}$, M. L. Ahnen ${ }^{a}$, M. Balbo ${ }^{c}$, M. Bergmann ${ }^{d}$, A. Biland ${ }^{a}$, C. Bockermann ${ }^{e}$, \\ T. Bretz ${ }^{a}$, K. A. Brügge ${ }^{b}$, D. Dorner ${ }^{d}$, S. Einecke ${ }^{b}$, J. Freiwald ${ }^{b}$, C. Hempfling ${ }^{d}$, \\ D. Hildebrand ${ }^{a}$, G. Hughes ${ }^{a}$, W. Lustermann ${ }^{a}$, K. Mannheim $^{d}$, K. Meier $^{d}$, S. Müller ${ }^{a}$, \\ D. Neise ${ }^{a}$, A. Neronov ${ }^{c}$, M. Nöthe ${ }^{b}$, A.-K. Overkemping ${ }^{b}$, A. Paravac ${ }^{d}$, F. Pauss $^{a}$, \\ W. Rhode ${ }^{b}$, F. Temme ${ }^{b}$, J. Thaele ${ }^{b}$, S. Toscano ${ }^{c}$, P. Vogler ${ }^{a}$, R. Walter ${ }^{c}$, and \\ A. Wilbert ${ }^{d}$ \\ E-mail: jens.bussetu-dortmund.de
}

${ }^{a}$ ETH Zurich, Institute for Particle Physics

Otto-Stern-Weg 5, 8093 Zurich, Switzerland

${ }^{b}$ TU Dortmund, Experimental Physics 5

Otto-Hahn-Str. 4, 44221 Dortmund, Germany

${ }^{c}$ University of Geneva, ISDC Data Center for Astrophysics

Chemin d'Ecogia 16, 1290 Versoix, Switzerland

${ }^{d}$ Universität Würzburg, Institute for Theoretical Physics and Astrophysics

Emil-Fischer-Str. 31, 97074 Würzburg, Germany

${ }^{e}$ TU Dortmund, Lehrstuhl für künstliche Intelligenz.

Otto-Hahn-Str. 12, 44221 Dortmund, Germany

The First G-APD Cherenkov Telescope (FACT) is the first operational telescope of its kind, employing a camera equipped with silicon photon detectors (G-APD aka. SiPM). SiPMs have a high photon detection efficiency (PDE), while being more robust to bright light conditions than the commonly used photo-multiplier tubes. This technology has allowed us to increase the duty cycle beyond that of the current generation of imaging air Cherenkov telescopes. During the last four years, the operation of FACT has proven that SiPMs are a suitable photon detectors for an application in the field of ground-based gamma-ray astronomy.

Nevertheless, it has been argued that crosstalk, after-pulses and dark counts are the main drawback of SiPMs, as these effects produce photon-like signals that would add up the signal background. Consequently, it is necessary to understand their impact on the analysis of data from FACT. In this contribution, we will show the current status of a study about the influence of different settings of crosstalk and dark counts on the performance of FACT. For that purpose, Monte Carlo simulations are used and compared to the actual data from the SiPM camera of FACT.

The 34th International Cosmic Ray Conference,

30 July- 6 August, 2015

The Hague, The Netherlands

\footnotetext{
${ }^{*}$ Speaker.
} 


\section{Introduction}

The camera of the first G-APD Cherenkov Telescope (FACT) is equipped with 1440 pixels, each a silicon photo multiplier (SiPM). During the last four years, the FACT collaboration has demonstrated the successful application of silicon photon detectors (G-APDs aka. SiPMs) to imaging atmospheric Cherenkov telescopes (IACTs) [2].

In the past, optical crosstalk, after-pulses and dark counts were considered to be the main drawback of SiPMs. FACT has been able to show, that these effects have a minor influence on the data of an IACT. This is discussed in more detail in [3].

In the last two years, the FACT collaboration has made a lot of progress in bringing data and Monte Carlo simulations in agreement. Recent comparisons of data and simulation on raw data and image parameter level, indicate that the properties of the FACT camera are well represented by the simulation. This allows for simulating different settings of the SiPM's crosstalk probability and dark count, in order to investigate their impact on the data analysis chain of FACT in more detail.

The Sensors The FACT camera detects photons by use of SiPMs (type: Hamamatsu MPPC S10362-33-50C). These semiconductor detectors consist of several Geiger-mode avalanche diodes (G-APDs) that are operated above their breakdown voltage. An incoming photon induces a complete discharge of the affected diode and produces a well defined signal waveform. Each diode is capable of detecting a single photon. The resulting signal of a whole SiPM is a superposition of the individual G-APD signals. The number of detected photons (hereafter called photon charge) can consequently be determined from the multiplicity of single photon signals.

However, there are certain phenomena that add up the signal background. For example, optical crosstalk and dark counts produce avalanches that are mostly indistinguishable from photon induced signals. Dark counts are avalanches that inherit from thermal excitation of electron-hole pairs in the semiconductor material. This process causes a uniformly distributed occurrence of avalanches in G-APDs in time. Each of these avalanche processes actually emits a random number photons itself. These photons can reach neighboring G-APDs and may trigger additional avalanches there. This effect is known as optical crosstalk and can be considered in coincidence with the breakdown in the first diode. Accordingly, the resulting signal shows an exaggerated photon multiplicity concerning incoming photons. This may cause a systematic bias on the photon charge extraction $[2,8]$.

The SiPMs used by FACT, show a crosstalk probability of $\approx 13 \%$ and a dark count rate of a few MHz. The night-sky background rate, in turn, can variate from tens of $\mathrm{MHz}$ up to more than $10 \mathrm{GHz}$ during full moon. Compared to this rate the dark count rate is negligible [2, 3].

In order to investigate the impact of different properties for dark counts and crosstalk, simulations of $\gamma$-ray induced showers were used in combination with the simulation of the FACT system (Reflector and Camera). A brief overview of this simulation chain is given in the following section.

\section{Simulation}

The basis of this study is a simulation of $N_{0} \approx 12 \cdot 10^{6}$ gamma showers, which are simulated in an energy range of $E=[200 \mathrm{GeV}, 50 \mathrm{TeV}]$ with a spectral index of $\Gamma=-2.7$. The night-sky background is simulated with a rate of $22 \mathrm{MHz}$. The showers are simulated with the extensive air 
shower simulation CORSIKA [7]. Afterwards, the simulated showers are fed into the reflector and camera simulation CERES, which was developed for the simulation of FACT. CERES is part of the ROOT based framework MARS CHEOBS [5]. Among the simulation of e.g. reflector, trigger and readout electronics, a dedicated simulation of the SiPMs and their properties is implemented. Developments in CERES are constantly verified by comparison with actual data, yielding a well fitting match of data and Monte Carlo simulations, as presented in the following.

\section{Comparison of Data and Monte Carlo}

Through the use of dedicated calibration runs, which are taken with a closed lid and a random trigger of $25 \mathrm{~Hz}$, a photon charge spectrum of dark count signals can be generated (see Figure 1, black curve). The black distribution shows the extracted charges, which are determined by integration over a fixed window of $15 \mathrm{~ns}$ of a candidate pulse. The spectrum is mostly influenced by dark counts, after-pulses and crosstalk events. Comparing its morphology allows for verifying a proper simulation of these effects. Variations of the extracted charge, mostly, due to afterpulses and electronic noise, as well as, gain differences of single G-APD cells, fold into the width of the peaks. Each peak represents a multiplicity of coincident breakdowns. Crosstalk can be identified by the slope of the amplitudes of the peaks with different photon multiplicity [3].
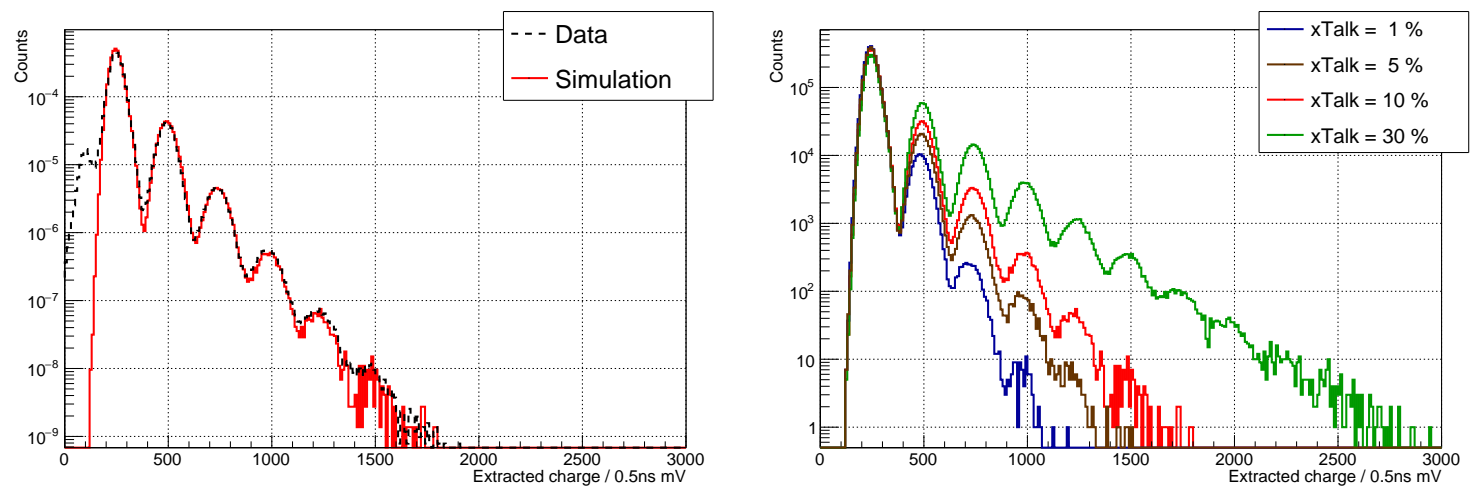

Figure 1: Examples for the spectrum of charges extracted from events induced by dark counts in combination with crosstalk and after-pulses, in the absence of night-sky background photons. The comparison of a simulated spectrum (left, red curve) and an example spectrum from data (left, black curve) shows a good agreement. The total area under the curve is scaled to one. An additional peak from artifacts of the readout electronics is visible in the data at about $100 \cdot 0.5 \mathrm{~ns} \cdot \mathrm{mV}$. These artifacts are not simulated and therefor not visible in the red distribution. The effect of different simulations of the crosstalk probability to the morphology of the spectra is displayed in the right picture. All four spectra were simulated with the same dark count rate of $5 \mathrm{MHz}$.

A simulated dark count spectrum is depicted by the red distribution in Figure 1 (left plot). The distributions of data and simulated events are in good agreement. The additional peak in the data at about $100 \cdot 0.5 \mathrm{~ns} \cdot \mathrm{mV}$ originates from artifacts of the readout electronics that are not simulated. Consequently, a correct simulation of the SiPM's properties can be considered.

For the following study of dark counts and crosstalk, their properties are variated separately in the simulation. The simulated conditions were chosen to be approximatively in the range of 
current generation SiPMs. The dark count rate is varied between $100 \mathrm{kHz}$ and $10 \mathrm{MHz}$. Crosstalk is simulated from $1 \%$ to $30 \%$. An example for the impact of different crosstalk probabilities to the dark count spectrum is given in Figure 1. The change of the slope of the peak heights is rising with the simulated crosstalk probability.

\section{Impact on the Analysis Chain}

The study presented here was performed using the analysis framework FACT-Tools [4, 6], which features the processing of raw data e.g. in (compressed) Fits format [1] up to the parameterization of the shower image by use of i.e. Hillas parameters. The behavior of certain analysis steps depending on the properties described in section 3, will be discussed, by focusing on the photon charge extraction, the image cleaning and the distribution of exemplary image parameters. In case of the image cleaning, the cleaning levels were fixed to the same values for all simulated properties.

Photon Charge Extraction The extraction of the photon charge is performed with the already mentioned extraction algorithm [3]. Extracted signals are normalized by the charge of a single avalanche and measured in photon equivalents (p.e.). The performance of the charge extractor is determined by computation of the relative divergence $C_{d i v}$ of simulated photon charge $C_{s i m}$ and extracted photon charge $C_{e x t r}$, given by

$$
C_{d i v}=\frac{C_{e x t r}-C_{s i m}}{C_{s i m}} .
$$

With the distribution of $C_{d i v}$ for certain quantities of $C_{s i m}$, we determine the bias and resolution of the extractor from the distribution's mean and standard deviation. Figure 2 and 3 illustrate the performance of the charge extractor for different quantities of simulated shower photons.
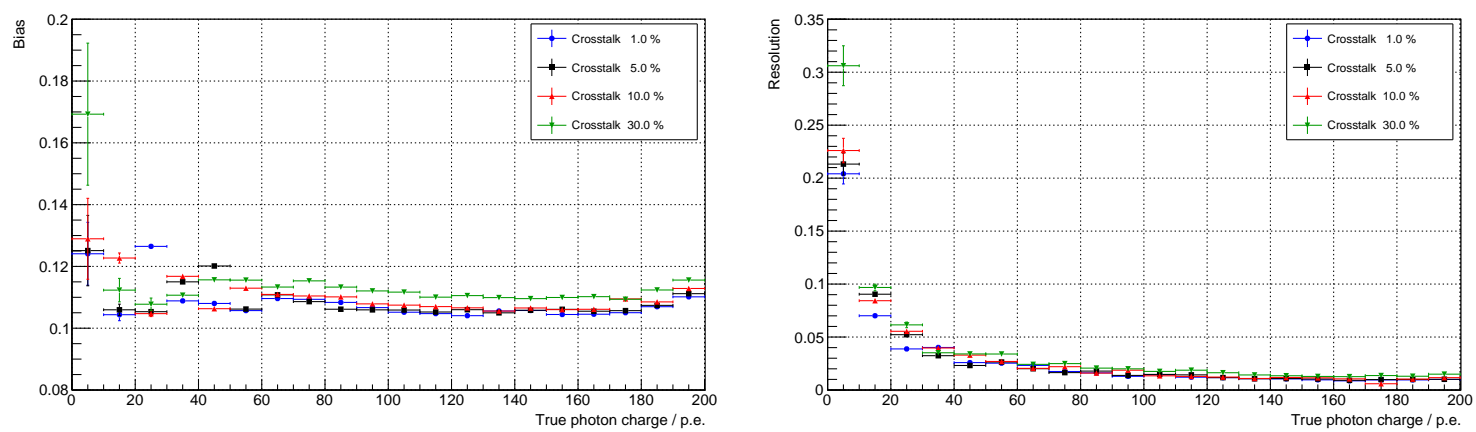

Figure 2: Dependency of the different crosstalk probabilities to the bias (left) and the resolution (right) of the photon charge extraction algorithm, performed on the pixels of cleaned shower images. The cleaning levels were fixed to the same values for all simulated properties.

As shown in Figure 2, different crosstalk probabilities seem to have a minor effect on the extractions performance. The bias of all four settings does not deviate by more than $2 \%$. Only in the regime of 0 p.e. to 10 p.e., significant deviations are visible. This can be explained by an extraction of dark counts and night-sky background photons with increased charge due to crosstalk. The resolution is mostly influenced in the low p.e. regime, since high crosstalk probabilities increase 
the rate of accidentally extracted background photons. Above $\approx 50$ p.e. the difference in resolution is negligible.
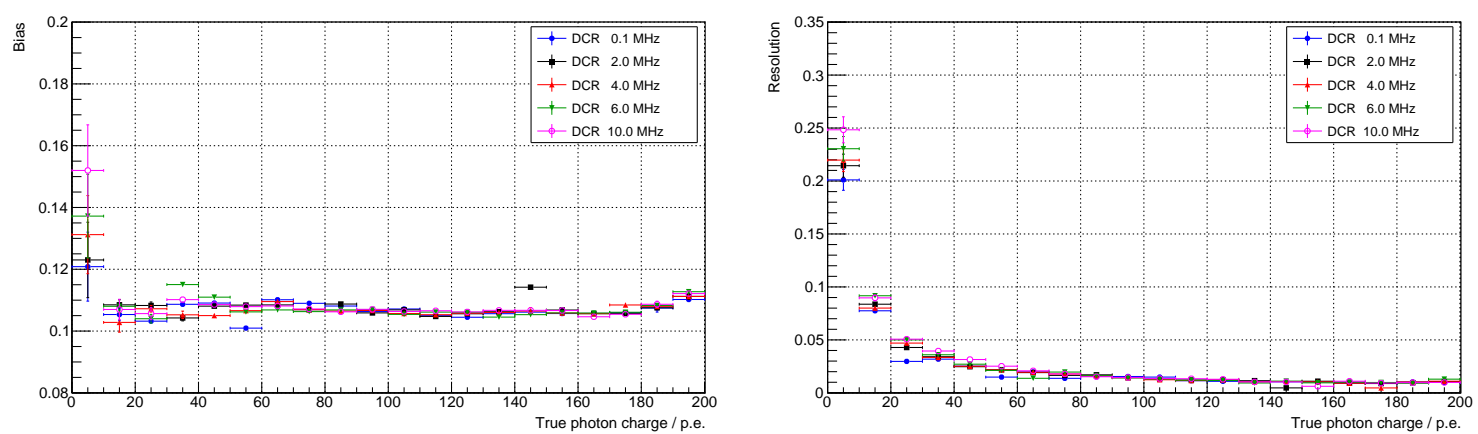

Figure 3: Dependency of the different dark count rates (DCR) to the bias (left) and the resolution (right) of the photon charge extraction algorithm, performed on the pixels of cleaned shower images. The cleaning levels were fixed to the same values for all simulated properties.

The simulation of different dark count rates shows comparable results (see figure 3). Again the bias is mostly affected for low photon quantities, which again can be explained by accidental extraction of noise photons. Above 10 pe no significant influence of the dark count rate is visible. Nevertheless, decreasing the dark count rate to $\approx 100 \mathrm{kHz}$ would improve the resolution below 50 p.e. Above this quantity the differences in resolution are negligible.
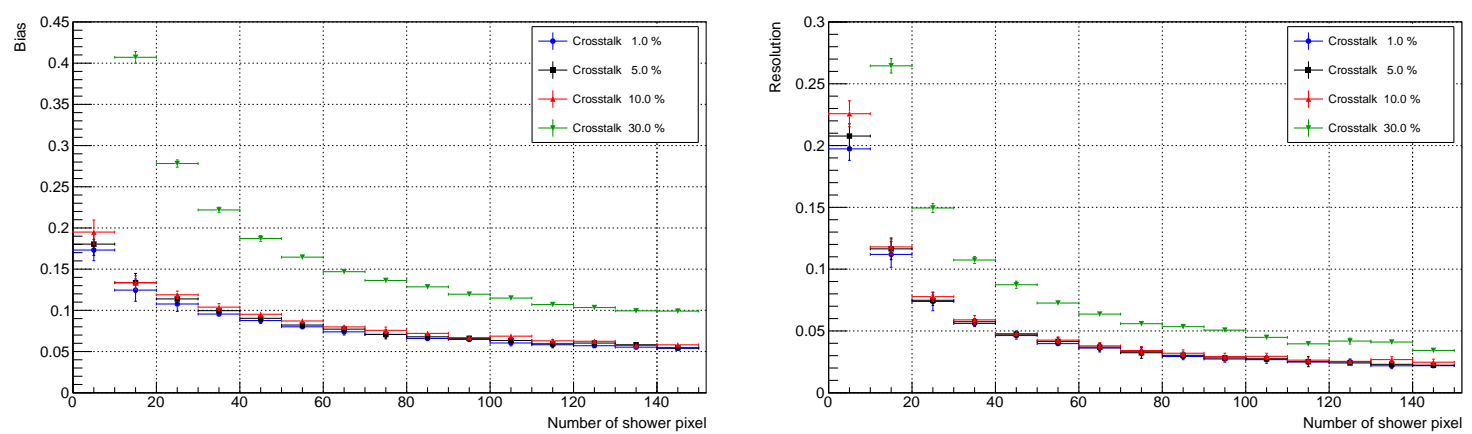

Figure 4: Dependency of different simulations of crosstalk probabilities to the bias (left) and the resolution (right) of the number of selected shower pixels, that survived the image cleaning. The cleaning levels were fixed to the same values for all simulated properties.

Cleaning The current analysis features a two level time-neighbor-cleaning. A cluster of pixels is identified as shower if the core pixels contain more than 5.5. p.e. while the neighboring pixels contain at least 3 p.e. The time difference of neighboring pixels is restricted to be less than $5 \mathrm{~ns}$. Equation 4.1 is used to determine the divergence of the number of pixels with Cherenkov photons (Monte Carlo truth) to the extracted number of shower pixels. With these values, the bias and the resolution of the cleaning algorithm are computed. Figure 4 and 5 show the performance of the cleaning algorithm. Bias and resolution are clearly depending on the crosstalk probability, as presented in Figure 4. With less crosstalk, fewer pixels are capable of exceeding the cleaning 

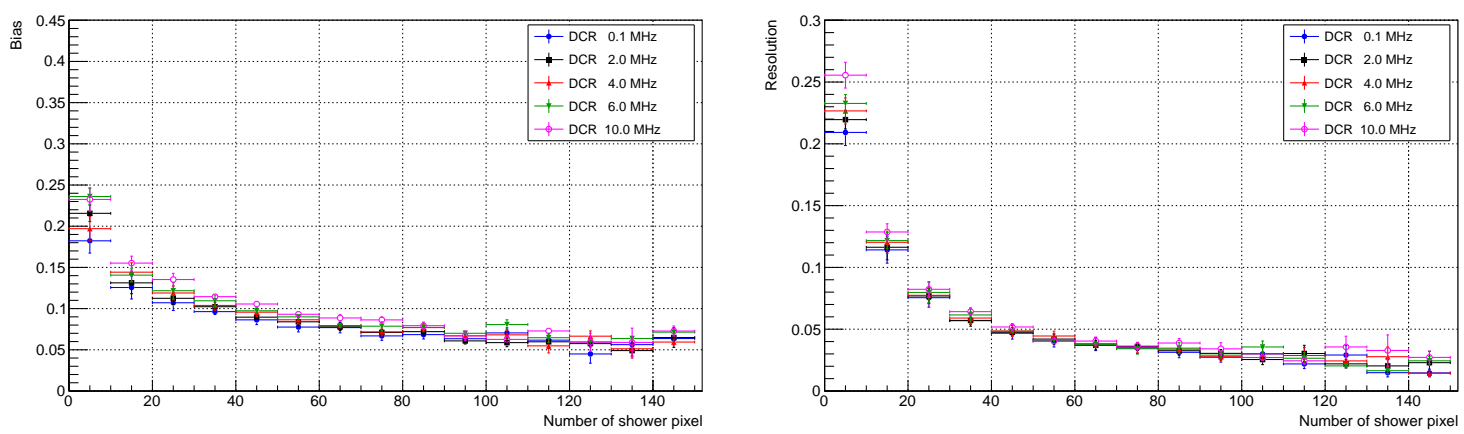

Figure 5: Dependency of the different simulations of dark count rates (DCR) to the bias (left) and the resolution (right) of the number of selected shower pixels, that survived the image cleaning. The cleaning levels were fixed to the same values for all simulated properties.

levels. Consequently the bias is lower for a decreased crosstalk probability. Below $10 \%$ crosstalk probability, the gain of performance is negligible within the uncertainties.

As depicted in Figure 5, a reduction of the dark count rate leads to a reduction of the cleaning bias. Especially the bias of small showers (less than 10 pixels) can be reduced. The same accounts for the resolution of the cleaning algorithm. Between a dark count rate of $100 \mathrm{kHz}$ and $10 \mathrm{MHz}$ the resolution for small showers can be improved by $\approx 5 \%$. In turn, the classification of pixels in large showers shows no significant improvement.
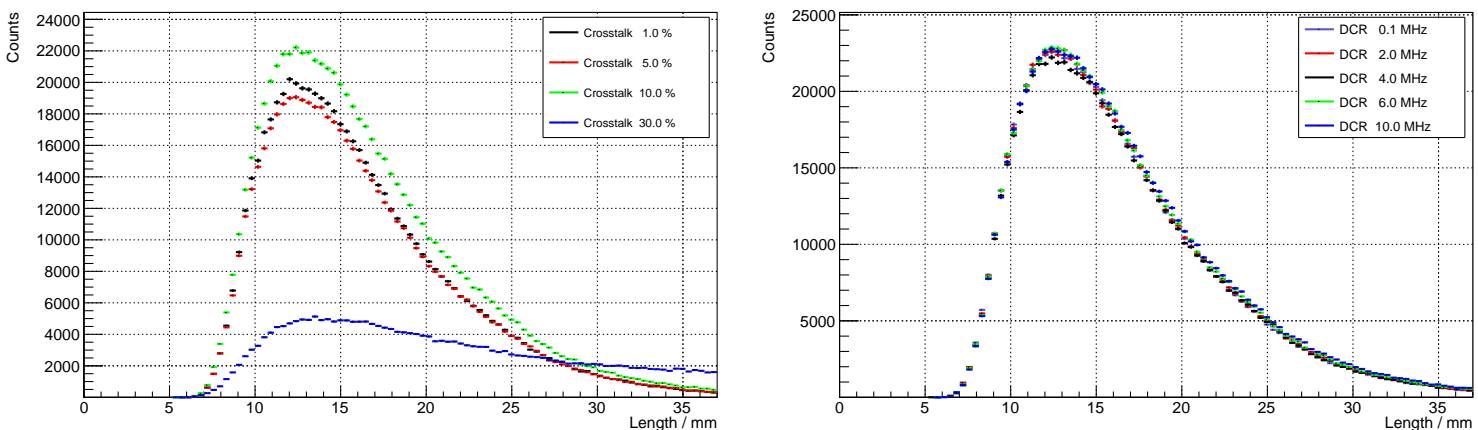

Figure 6: Distribution of the shower length for different crosstalk probabilities (left) and different dark count rates (DCR, right), as example for the impact on the shower image parameterization. All distributions inherit from the same simulated observation time. The cleaning levels were fixed to the same values for all simulated properties, accordingly the distributions are dependent on the chosen thresholds.

Image parameters The influence on the parameterization of the shower images is discussed, by the example of the shower length distribution (Length). The according distributions for crosstalk and dark counts are displayed in Figure 6. Large crosstalk probabilities cause the Length distribution to be washed out. More background photons contribute to the shower image and less showers survive the cleaning. This is also an effect of the fixed cleaning levels. With decreasing crosstalk, the distribution gets more distinct. Nevertheless, the improvement between $10 \%$ and $1 \%$ crosstalk is small. With a crosstalk probability of $10 \%$ the trigger rate is the highest, since the 
current cleaning was tuned on data with a comparable crosstalk probability. For the dark count rate, no hint for a dependency of Length and the simulated rates was found. In the course of this study, also other image parameters were investigated e.g. Width and Area. They affirm the effects registered for the Length.

Energy Distribution The distribution of the Monte Carlo energy after image cleaning is depicted in Figure 7. The variation of the dark count rate shows no impact on the remaining energy distribution, since the distribution of random photons is dominated by the night-sky background rate. However, crosstalk shows a clear impact. An increasing crosstalk causes more showers to be discarded by the cleaning. This lowers the trigger rate after cleaning significantly. At the same time, relatively more showers with small energies survive the cleaning and the threshold seems to be shifted to lower energies. However, less noise photons are cleaned and distort the reconstructed image parameters. This, for example, corrupts the energy reconstruction of the affected showers, since misclassified shower pixels cause a bias on the energy reconstruction and impair its resolution.
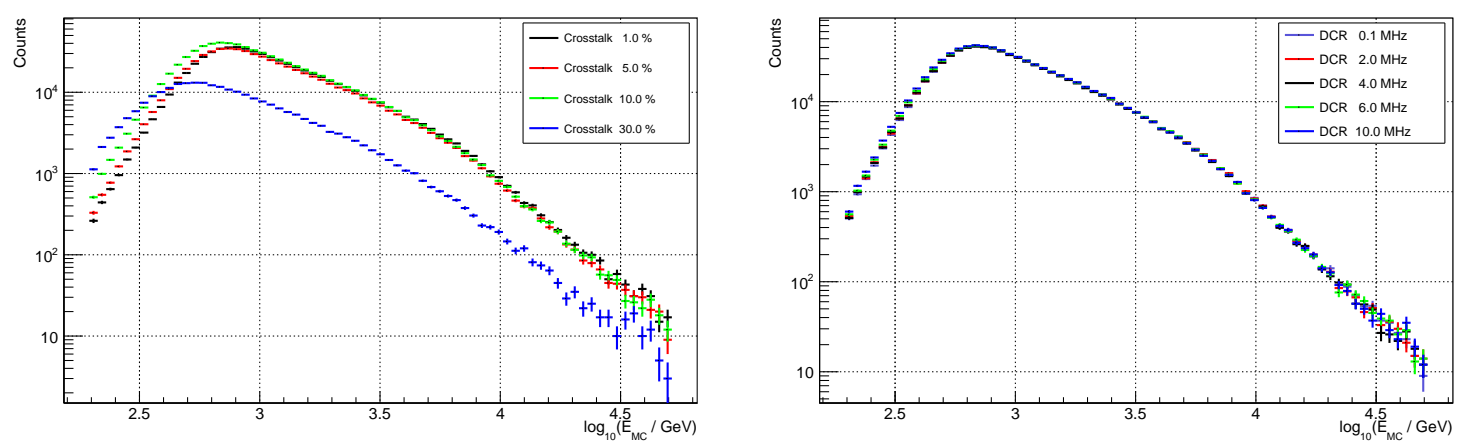

Figure 7: Monte Carlo energy distribution of simulated gamma events after image cleaning, with variation of the crosstalk probability (left) and the dark count rate (DCR, right). All distributions inherit from the same simulated observation time. The cleaning levels were fixed to the same values for all simulated properties. For large crosstalk probabilities more showers with smaller energies can survive the cleaning, but less showers are triggered. Therefore the energy threshold seems to be shifted to lower energies, while the energy reconstruction is corrupted by noise photons.

\section{Conclusion}

The properties of the SiPMs used for FACT already show a very good performance in data analysis. The bias of photon charge extraction and image cleaning is $\approx 10 \%$. Regarding the SiPMs used in FACT and the current generation of devices, the bias and the resolution of the shower reconstruction are promising to differ by only a few percent. Larger differences are only seen in the range of faint pixels (below 10 p.e.). With the simulated settings and the used cleaning levels, a significant impact on the examined image parameters and the energy distribution, was only registered for variations of the crosstalk probability. This effect was determined to be rather small for low crosstalk probabilities below $10 \%$, where the SiPMs of FACT are operating, but large for high crosstalk probabilities. 
Improvements of the SiPM's dark count and crosstalk behavior will mainly affect the extraction of pixels with small p.e. The benefit will be a gain of precision in the classification of small showers and the classification of edge pixels. This will allow for lowering the levels for the image cleaning, which will also lower the energy threshold of the detector. Thus, a modification of cleaning levels will be considered in a follow-up study to the presented work. A further investigation of the impact of crosstalk regarding the energy reconstruction is in preparation.

Acknowledgment The important contributions from ETH Zurich grants ETH-10.08-2 and ETH-27.12-1 as well as the funding by the German BMBF (Verbundforschung Astro- und Astroteilchenphysik) and HAP (Helmoltz Alliance for Astroparticle Physics) are gratefully acknowledged. We are thankful for the very valuable contributions from E. Lorenz, D. Renker and G. Viertel during the early phase of the project. We thank the Instituto de Astrofisica de Canarias allowing us to operate the telescope at the Observatorio del Roque de los Muchachos in La Palma, the Max-Planck-Institut für Physik for providing us with the mount of the former HEGRA CT 3 telescope, and the MAGIC collaboration for their support. Part of this work is supported by Deutsche Forschungsgemeinschaft (DFG) within the Collaborative Research Center SFB 876 "Providing Information by Research-Constrained Analysis", project C3.

\section{References}

[1] M. L. Ahnen et al. Data compression for the First G-APD Cherenkov Telescope. In: ArXiv e-prints (June 2015). arXiv: 1506.06045 [astro-ph.IM] .

[2] H. Anderhub et al. Design and operation of FACT - the first G-APD Cherenkov telescope. In: JINST 8.06 (2013), P06008.

[3] A. Biland et al. Calibration and performance of the photon sensor response of FACT - the first G-APD Cherenkov telescope. In: JINST 9.10 (2014), P10012.

[4] C. Bockermann et al. Online Analysis of High-Volume Data Streams in Astroparticle Physics. In: Machine Learning: ECML 2015, Industrial Track. Springer Berlin Heidelberg, 2015.

[5] T. Bretz and D. Dorner. MARS - CheObs goes Monte Carlo. In: Proceedings of the 31st ICRC. 2009.

[6] K. Brügge et al. FACT-Tools: Streamed Real-Time Data Analysis. In: these proceedings. 1135. 2015.

[7] D. Heck et al. CORSIKA: A Monte Carlo Code to Simulate Extensive Air Showers," Report FZKA 6019, Forschungszentrum Karlsruhe. Tech. rep. 1998.

[8] D. Renker. Geiger-mode avalanche photodiodes for Cherenkov detectors. In: Journal of Instrumentation 5.01 (2010). 